LAXTON, R. R.

Math. Zeitschr. 83, 8-17(1964)

\title{
Prime ideals and the ideal-radical of a distributively generated near-ring
}

By

\author{
R. R. LAXTON
}

The concepts of a prime ideal of a distributively generated (d.g.) nearring $R$, a prime d.g. near-ring and an irreducible $R$-group are introduced ${ }^{1}$ ). The annihilating ideal of an irreducible $R$-group with an $R$-generator is a prime ideal. Consequently we define a prime ideal to be primitively prime if it is the annihilating ideal of such an $R$-group, and a d.g. near-ring to be a primitively prime near-ring if it acts faithfully on such a group. The intersection of all the primitively prime ideals of a d.g. near-ring is called the ideal-radical; this ideal contains all the nilpotent ideals of the near-ring and a relationship between it and the quasi-radical of the near-ring is established.

In section 2 we consider d.g. near-rings $R$ which satisfy the descending chain condition for left $R$-modules. In this case, the ideal-radical is nilpotent. Any non-zero prime d.g. near-ring is a primitively prime d.g. near-ring. All irreducible $R$-groups with an $R$-generator of a non-zero prime d.g. near-ring $R$ are shown to be isomorphic to the finite number of direct summands of the group $R^{+}-N$, where $N$ is the quasi-radical of $R$. If $R$ has finite order, then it has, to within an isomorphism, but one faithful representation on an irreducible $R$-group with an $R$-generator and all its irreducible $R$-groups with $R$-generators are homomorphic images of $R$-subgroups of this group.

In section 3, a number of equivalent conditions is given for a d.g. near-ring to have a nilpotent radical. One of them is that all its proper prime ideals are maximal ideals. In section 4, we construct an example of a finite d.g. near-ring whose radical is not nilpotent and whose quasi-radical is not an ideal.

\section{Definitions and general properties}

A near-ring $R$ is a system with two binary operations, addition and multiplication, such that:

(i) The elements of $R$ form a group $R^{+}$under addition.

(ii) The elements of $R$ form a semi-group under multiplication.

(iii) $(x+y) z=x z+y z$, for all $x, y, z \in R$.

1) The notation in this paper is different from that adopted in the two previous papers [7] and [8]. What was previously called an irreducible $R$-group is now called a minimal $R$-group. The term irreducible $R$-group is given another and more general meaning in this paper (see section 1). 
If $S$ is a multiplicative semi-group contained in $R$, whose elements generate $R^{+}$ and satisfy

(iv) $s(x+y)=s x+s y$,

we say that $R$ is generated by the distributive semi-group $S$. A near-ring $R$ which is generated by some distributive semi-group is said to be distributively generated.

Throughout this article, we shall mean by a d.g. near-ring a d. g. near-ring with an identity element which will be denoted by $e$ and $R$ will always denote such a near-ring. The symbol $S$ stands for some distributive semi-group generating $R$. It will always be assumed that $S$ contains $e$; this imposes no further structural restriction on $R$.

An $(R, S)$-group $\Omega$ is given by an additive group, the additive notation not to imply commutativity, together with a mapping $(x, w) \rightarrow x w$ of $R \times \Omega$ into $\Omega$ such that

(i) For all $x, y \in R$ and all $w \in \Omega,(x+y) w=x w+y w$.

(ii) For all $x, y \in R$ and all $w \in \Omega,(x y) w=x(y w)$.

(iii) For all $s \in S$ and $w_{1}, w_{2} \in \Omega, s\left(w_{1}+w_{2}\right)=s w_{1}+s w_{2}$.

(iv) For all $w \in \Omega, e w=w$.

If the particular semi-group $S$ occurring in this definition does not need to be specified, we simply speak of an $R$-group (see [5]).

The zero 0 of $R^{+}$is a two-sided annihilator of $R([2], 1.1,1.5)$. The zero of an additive group $\Omega$ will be denoted by $0_{\Omega}$, or, if no confusion is possible, simply by 0 . If $\Omega$ is an $R$-group, then $x 0_{\Omega}=0_{\Omega}$, for all $x \in R([3], 1.4)$.

A faithful $R$-group $\Omega$ is an $R$-group such that if $x \in R$ and $x w=0$ for all $w \in \Omega$, then $x=0$. A minimal $R$-group is a non-zero $R$-group which contains no $R$-groups as proper, non-zero sub-groups. A primitive d.g. near-ring $R$ is a d.g. near-ring which has a faithful representation on a minimal $R$-group (see $[7]$ ).

A homomorphism $\Phi$ of an $R$-group $\Omega$ into another $R$-group is called an $R$-homomorphism if $\Phi(x w)=x\left(\Phi_{w)}\right.$, for all $x \in R$ and $w \in \Omega$.

A sub-group a of $R^{+}$is a left (right) $R$-module of $R$ if $x y \in a(y x \in \mathfrak{a})$ for all $x \in R$ and $y \in a$. A left $R$-module that is also a right $R$-module is a two-sided $R$-module. A left (right, two-sided) ideal is a left (right, two-sided) $R$-module that is also normal in $R^{+}$. A two-sided ideal is simply called an ideal. Left $R$-modules are the $R$-subgroups of $R^{+}$. Left ideals are precisely the kernels of $R$-homomorphisms of $R^{+}$, and ideals are precisely the kernels of the nearring homomorphisms of $R([2], 1.3 .3,[3], 2.1 .4)$.

Definition 1. An ideal $p$ in a d.g. near-ring $R$ is called a prime ideal if and only if whenever $\mathfrak{a}$ and $\mathfrak{b}$ are ideals of $R$ and $\mathfrak{a} \mathfrak{b} \leq \mathfrak{p}$, then either $\mathfrak{a}$ or $\mathfrak{b}$ is contained in $p^{2}$ ). A d.g. near-ring whose zero ideal is prime is called a prime d.g. near-ring.

2) If $\mathfrak{a}_{1}, \ldots, \mathfrak{a}_{r}$ are subsets of $R$, then $\mathfrak{a}_{1} \mathfrak{a}_{2} \ldots \mathfrak{a}_{r}$ denotes the additive group generated by all elements of the form $a_{1} a_{2} \ldots a_{r}$, where $a_{i} \in \mathfrak{a}_{i}, i=1, \ldots, r$. If $\mathfrak{a}$ is a subset of $R$, we put $\mathfrak{a}^{n}=\mathfrak{a} \mathfrak{a} \ldots \mathfrak{a}$ ( $n$ times). We say that the set $\mathfrak{a}$ of $R$ is nilpotent if $\mathfrak{a}^{n}=(0)$ for some positive integer $n$. 
Lemma. If $\mathfrak{a}_{1}, \ldots, \mathfrak{a}_{\gamma}$ are ideals and $\mathfrak{p}$ is a prime ideal of $R$, then $\mathfrak{a}_{1} \mathfrak{a}_{2} \ldots \mathfrak{a}_{r} \subseteq p$ implies that $\mathfrak{a}_{i} \leqq p$ for some $i$.

Proot. If a set $a$ is contained in the ideal $p$, then so is the least ideal of $R$ containing $a$. We denote this ideal by $\bar{a}$. Hence if $a_{1} a_{2} \ldots a_{n}$ is contained in $p$ so is the ideal $\overline{a_{1} \mathfrak{a}_{2} \ldots \mathfrak{a}_{n n}}$. Now $\left(\overline{\mathfrak{a}_{1} \ldots \mathfrak{a}_{n-1}}\right) \mathfrak{a}_{n}$ is a product of two ideals and

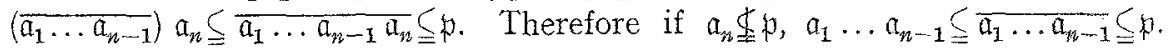
Repeating this argument gives the required result.

Definition 2. A non-zero $R$-group is called an irreducible $R$-group if it possesses no proper, non-zero, normal $R$-subgroups ${ }^{3}$ ).

In the following, we shall be concerned with irreducible $R$-groups $\Omega$ which possess an $R$-generator, that is, an element $w$ in $\Omega$ such that $R w=\Omega$. Such groups will be called cyclic irreducible $R$-groups. It is clear that a minimal $R$-group is a cyclic irreducible $R$-group.

Proposition 1. If $\Omega$ is a cyclic irreducible $R$-group, then the annihilating ideal $(0: \Omega)$ is a prime ideal of $\left.R^{4}\right)$.

Proot. The ideal $(0: \Omega)$ consists of all those $x \in R$ such that $x w=0$ for all $w \in \Omega$. Let $\mathfrak{a}$ and $\mathfrak{b}$ be two ideals of $R$ such that $\mathfrak{a} \$(0: \Omega)$ and $\mathfrak{b} \$(0: \Omega)$, and $w$ an $R$-generator of $\Omega$. Then the $R$-group $b \Omega=\mathfrak{b}(R w)=(\mathfrak{b} R) w=\mathfrak{b} w$ is not the zero sub-group of $\Omega$. If $w^{\prime} \in \Omega$, then there exists an element $z \in R$ such that $w^{\prime}=z w$ and so $w^{\prime}+b w-w^{\prime}=z w+b w-z w=(z+b-z) w \in b w$, for all $b \in \mathfrak{b}$. Thus $b$ w is a non-zero normal $R$-subgroup of $\Omega$; hence $b w=\Omega$. Now the group $(\mathfrak{a} \mathfrak{b}) \Omega \supseteqq(\mathfrak{a} b) w=\mathfrak{a}(\mathfrak{b} w)=\mathfrak{a} Q$ is a non-zero subgroup of $\Omega$. Therefore $(\mathfrak{a} \mathfrak{b}) \$(0: \Omega)$ and this proves that the ideal $(0: \Omega)$ is prime.

Definition 3. A d.g. near-ring $R$ which has a faithful representation on a cyclic irreducible $R$-group is called a primitively prime d.g. near-ring. An ideal $p$ of a d.g. near-ring $R$ is called a primitively prime ideal if $R / p$ is a primitively prime d.g. near-ring.

The above proposition shows that a primitively prime d.g. near-ring is a prime d.g. near-ring and a primitively prime ideal is a prime ideal. A primitively prime ideal is a proper ideal.

Proposition 2. An ideal $\mathfrak{p}$ in $R$ is primitively prime if and only if $\mathfrak{p}=(\mathfrak{l}: R)$, where $Y$ is a maximal left ideal of $R$.

Proof. Let $p=(\mathfrak{l}: R)$; then $R / p$ acts faithfully on the irreducible $R / \mathfrak{p}$-group $R^{-}-1$. The image of the identity element $e$ of $R$ under the homomorphism

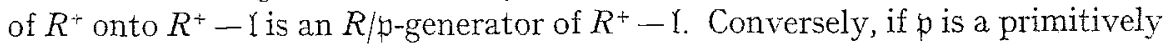
prime ideal, let $\Omega$ be a faithful, irreducible $R / p$-group with an $R / p$-generator $w$, say. Then $\Omega$ is an $R$-group, $R w=\Omega$ and the mapping of $R^{+}$onto $\Omega$ given by $x \rightarrow x w$, for all $x \in R$, is an $R$-homomorphism. Hence $R^{+}-(0: w) \hat{=} \Omega ;(0 ; w)$ is a maximal left ideal of $R$ and $\mathfrak{p}=((0 ; w): R)$.

If $\mathfrak{a}$ is a subset of $R$ and $\Delta$ is a subset of an $R$-group $\Omega$, then $a A$ denotes the group generated by all elements of the form $a w$, where $a \in \mathfrak{a}$ and $w \in A$.

${ }^{3}$ ) See footnote ${ }^{1}$ ).

4) If $\Delta_{1}$ and $\Delta_{2}$ are two subsets of an $R$-group, then $\left(\Delta_{1}: \Delta_{2}\right)$ denotes the set of elements of $R$ which map $\Delta_{2}$ into $\Delta_{1}$. We note that if $\llbracket$ is a left ideal of $R$, then $(I: R)$ is an ideal of $R$ contained in $\mathfrak{l}$ which contains all ideals of $R$ in $\mathfrak{l}([2], 3.7 .1)$. 
The primitive ideals of $R$ are by definition the annihilating ideals of minimal $R$-groups (see $[8]$ ). Hence:

Proposition 3. The primitive ideals of a d.g. near-ring are primitively prime ideals and primitive d.g. near-rings are primitively prime d.g. near-rings.

Proposition 4. A prime ideal p of a d.g. near-ring $R$ contains all the nilpotent $i d e a l s$ of $R$. The factor near-ring $R / p$ has no non-zero nilpotent ideals.

Proot. If $\mathfrak{a}$ is an ideal and $\mathfrak{a} \mathfrak{a} \ldots \mathfrak{a} \subseteq \mathfrak{p}$, then $\mathfrak{a} \subseteq \mathfrak{p}$ by the lemma.

The intersection of all the primitive ideals of a d.g. near-ring $R$ is called the radical (if the near-ring has no primitive ideals, then the radical is taken to be the whole near-ring) and is equal to the intersection of all the maximal left ideals which are maximal left $R$-modules of $R$. The intersection of all the maximal left ideals of a d.g. near-ring is called the quasi-radical (see [8]). Any d.g. near-ring with an identity element contains maximal left ideals (which are proper left ideals). This is proved by an application of ZoRN's lemma. Therefore, by proposition 2, any d.g. near-ring with an identity element possesses primitively prime ideals.

Definition 4. The intersection of all the primitively prime ideals of a d.g. near-ring is called the ideal-radical of the near-ring.

Theorem 1. Let $N$ denote the quasi-radical of a d.g. near-ring $R$. The idealradical is the ideal $(N: R)$ which is contained in $N$. The ideal-radical contains all the nilpotent ideals of the near-ring.

Proot. Let $P$ denote the ideal-radical of $R, P$ is contained in the ideal $(\mathfrak{l}: R)$ for each maximal left ideal $\mathfrak{l}$ of $R$ by proposition 2 . Since $(\mathfrak{l}: R) \leqq \mathfrak{l}, P$ is contained in each maximal left ideal of $R$ and so in the intersection of all the maximaI left ideals of $R$, i.e., the quasi-radical $N$ of $R$. But $P$ is an ideal and

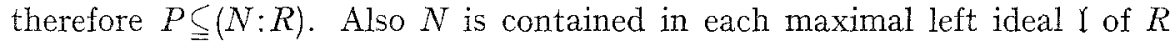
and hence $(\bar{N}: R) \leqq(\mathfrak{l}: R)$, for each maximal left ideal $\mathfrak{l}$ of $R$. Therefore $(N: R)$ is contained in the intersection of the ideals $(\mathfrak{l}: R)$ where $\mathfrak{l}$ is a maximal left ideal of $R$, i.e., $(N: R) \subseteq P$ by proposition 2. Therefore the ideal $(N: R)$ is the ideal-radical of $R$.

The last part of the theorem follows immediately from proposition 4.

We have the following situation for a d.g. near-ring $R$ : the radical contains the quasi-radical which contains the ideal-radical. The radical is an ideal (which may or may not be proper) containing all the nilpotent left $R$-modules of $R$. The quasi-radical is a proper left ideal containing all the nilpotent left ideals of $R$. The ideal-radical is an ideal containing all the nilpotent ideals of $R$. Further properties of these three "radicals" are obtained in the next section for any d.g. near-ring $R$ which satisfies the descending chain condition for left $R$-modules (see $[8]$ ).

\section{Further properties for d.g. near-rings which satisfy the descending chain condition}

Any d.g. near-ring $R$ which appears in this and the following section satisfies the descending chain condition (d.c.c.) for left $R$-modules. In this 
case, the primitive ideals of $R$ are maximal ideals and primitive d.g. near-rings are simple near-rings, that is, they possess no proper, non-zero ideals (see [7]). The restatement of proposition 3 is

Proposition 5. The maximal ideals of a d.g. near-ring $R$ which satisfies the d.c.c. for left $R$-modules are primitively prime ideals. If $R$ is a simple d.g. near-ring, then it is a primitively prime near-ring.

Theorem 2. Let $R$ be a d.g.near-ring satisfying the d.c.c. for left $R$-modules. The ideal-radical is a nilpotent ideal containing all the nilpotent ideals of $R$.

Proof. The quasi-radical of $R$ is nilpotent (see [8]) and so the ideal-radical must be nilpotent.

Corollary. $R$ has a non-zero nilpotent ideal if and only if its ideal-radical $P$ is non-zero. The near-ring $R / P$ has no non-zero, nilpotent ideals.

We consider the $R$-group $R^{+}-N$, where $N$ is the quasi-radical. The intersection of all the maximal left ideals of $R$ is $N$. Since $R$ satisfies the d.c.c. for left $R$-modules, there exists a finite number of distinct maximal left ideals $\mathfrak{l}_{1}, \ldots, \mathfrak{l}_{n}$ such that $\bigcap_{i=1}^{n} \mathfrak{l}_{i}=N$ and $\mathfrak{\Omega}_{k}=\bigcap_{\substack{i=1 \\ i \neq k}}^{n} \mathfrak{l}_{i} \neq N$ for any $k=1, \ldots, n$. In the canonical $R$-homomorphism of $R^{+}$onto $R^{+}-N$ let $\mathfrak{l}_{k}$ be mapped onto $\Omega_{n}$ and $\Omega_{k}$ onto $\Delta_{k}$ for all $k=1, \ldots, n$. Since $\bigcap_{i=1}^{n} Y_{i}=N$ it follows that $\bigcap_{i=1}^{n} \Omega_{i}=(0)$. Since the $\mathfrak{I}_{k}$ are maximal left ideals $R=\mathfrak{I}_{k}+\mathfrak{Q}_{k}$ and so $R^{+}-N=\Omega_{k} \oplus \Delta_{k}$ for $k=1, \ldots, n$. Also $\mathfrak{I}_{k} \geqq \Omega_{i}$ for all $j \neq k$ and $\mathfrak{I}_{k} \cap \Omega_{k}=N$ for all $k=1, \ldots, n$, therefore $\Omega_{k} \geqq \Delta_{j}$ for all $j \neq k$ and $\Omega_{k} \cap \Delta_{k}=(0)$ for all $k=1, \ldots, n$.

If $m \neq k, \Omega_{m}=\Omega_{m} \cap\left(R^{+}-N\right)=\Omega_{m} \cap\left(\Delta_{k} \oplus \Omega_{k}\right)=\Delta_{k} \oplus \Omega_{k} \cap \Omega_{m}$ by the modular law which holds for the lattice of normal subgroups of $R^{+}-N$. Hence $R^{+}-N=$ $\Omega_{m} \oplus \Delta_{m}=\left(\Delta_{k} \oplus \Omega_{k} \cap \Omega_{m}\right) \oplus \Delta_{m}=\Delta_{k} \oplus A_{m} \oplus \Omega_{k} \cap \Omega_{m}$.

If $n \neq m, \quad k, \quad \Omega_{k} \cap \Omega_{m}=\Omega_{k} \cap \Omega_{m} \cap\left(\Delta_{n} \oplus \Omega_{n}\right)=\Delta_{n} \oplus \Omega_{k} \cap \Omega_{m} \cap Q_{n}$ and hence $R^{+}-N=\Delta_{k} \oplus \Delta_{m} \oplus \Delta_{n} \oplus \Omega_{k} \cap \Omega_{m} \cap \Omega_{n}$. Since $\bigcap_{i=1}^{n} \Omega_{i}=(0)$ it follows that we obtain finally

$$
R^{+}-N=\Delta_{1} \oplus \cdots \oplus \Delta_{n},
$$

where $A_{k} \cong\left(R^{+}-N\right)-\Omega_{k} \cong R^{+}-\mathfrak{Y}_{k}$ for $k=1, \ldots, n$. From these isomorphisms it follows that the $\Delta_{k}$ are irreducible $R$-groups. If $e$ is mapped onto $\bar{e}$ under the canonical homomorphism of $R^{+}$onto $R^{+}-N$ and $\bar{e}=\bar{e}_{1}+\bar{e}_{2}+\cdots+\bar{e}_{n}$, $\bar{e}_{k} \in \Delta_{k}$, then $R \bar{e}_{k}=\Delta_{k}$ for $\left.k=1, \ldots, n^{5}\right)$. Thus the $\Delta_{k}$ are cyclic irreducible $R$-groups.

Let $\Omega$ be a cyclic irreducible $R$-group with an $R$-generator $w$, say. $R^{+}-(0: w) \subseteq \Omega$ and $(0: w)$ is a maximal left ideal. Thus $(0: w)$ contains $N$

5) We note that if $R$ is a d.g. near-ring and the ( $R, S)$-group $\Omega=\Omega_{1} \oplus \cdots \oplus \Omega_{n}$ is a direct sum of $R$-groups, then $x\left(w_{1}+\cdots+w_{n}\right)=x w_{1}+\cdots+x w_{n}$ for all $x \in R$, where $w_{k} \in \Omega_{k}$ for $k=1, \ldots, n$. 
and from (1) $R^{+}-(0: w)$ must be $R$-isomorphic to one of the $R$-groups $\left.\Delta_{k}^{6}\right)$. Hence

Theorem 3. Let $R$ be a d.g. near-ring satisfying the d.c.c. for left $R$-modules. Any cyclic irreducible $R$-group is $R$-isomorphic to one of the finite number of irreducible $R$-groups which appear in the direct sum decomposition (1) of $R^{+}-N$.

Now let $p$ be a proper prime ideal in $R$; put $\bar{R}=R / p$ and denote by $\bar{N}$ the quasi-radical of $\bar{R}, \bar{R}$ is a prime d.g. near-ring satisfying the d.c.c. for left $\bar{R}$-modules. The zero ideal $(\overline{0})$ of $\bar{R}$ is prime and so $\bar{R}$ contains no non-zero nilpotent ideals, in particular, $(\bar{N}: \bar{R})=(\overline{0})$. Hence $\bar{R}$ acts faithfully on the group $\bar{R}^{+}-\bar{N}$. By (1), $\bar{R}^{+}-\bar{N}=\bar{\Delta}_{1}+\cdots+\bar{\Delta}_{*}$, where the $\bar{\Delta}_{k}$ are cyclic irreducible $\bar{R}$-groups. The annihilating ideals $\left(\overline{0}: \overline{\Delta_{k}}\right)$ are primitively prime ideals and $\bigcap_{i=1}^{r}\left(\overline{0}: \bar{\Delta}_{k}\right)=(\overline{0})$. Therefore

$$
\left(\overline{0}: \bar{\Delta}_{1}\right)\left(\overline{0}: \bar{\Delta}_{2}\right) \ldots\left(\overline{0}: \bar{\Delta}_{r}\right) \leqq \bigcap_{i=1}^{r}\left(\overline{0}: \bar{\Delta}_{i}\right)=(\overline{0})
$$

and, since $(\overline{0})$ is a prime ideal, $\left(\overline{0}: \bar{\Delta}_{k}\right)=(\overline{0})$ for some $k$. In other words, $\bar{R}$ acts faithfully on some $\bar{A}_{k}$. Hence

Theorem 4. Let $R$ be a d.g. near-ring satisfying the d.c.c. for left $R$-modules. Any proper prime ideal of $R$ is a primitively prime ideal. If $R$ is a non-zero prime d.g. near-ring, then it is a primitively prime d.g. near-ring.

We end this section with a structure theorem for finite prime d.g. nearrings which gives a relationship between all the cyclic irreducible $R$-groups. To a certain extent it generalizes the theorem for simple d.g. near-rings which states that all the minimal $R$-groups of a simple d.g. near-ring $R$ are $R$-isomorphic (see [7]).

Theorem 5. Let $R$ be a non-zero finite, prime d.g. near-ring. All cyclic irreducible $R$-groups are $R$-homomorphic images of $R$-subgroups of a faithful, cyclic irreducible $R$-group. To within an isomorphism, $R$ has one, and only one, faithful, cyclic irreducible $R$-group.

Proof. $R$ is not the zero near-ring. Let $N$ be the quasi-radical of $R$. Then $R^{+}-N=\Delta_{1} \oplus \cdots \oplus \Delta_{n}$, where the $\Delta_{k}$ are irreducible $R$-groups with $R$-generators $\bar{e}_{k}, \bar{e}=\bar{e}_{1}+\cdots+\bar{e}_{n}$. From the previous theorem, $R$ acts faithfully on some $\Delta_{k}$, say $\Delta_{n}$. Denote by $I$ the set of all groups which are $R$-homomorphic images of $R$-subgroups of $\Delta_{n}$ and, by $I^{\prime}$, those groups of $I$ which are cyclic

$\left.{ }^{6}\right)$ We are using here the fact that an $R$-group is an $(R, S)$-group for some set $S$ of distributive elements of $R$. Thus an $R$-group is an operator group with $S$ as a set of operators. Since $S$ generated $R^{+}$a subgroup of an $(R, S)$-group is an $R$-subgroup if and only if it is an admissible subgroup for the set of operators $S$ and two $(R, S)$-groups are $R$-isomorphic if they are isomorphic as operator groups for the set of operators $S$. It follows that in the present case the decomposition (1) of $R^{+}-N$ provides us with the composition series $\Delta_{1} \oplus \cdots \oplus \Delta_{n}>\Delta_{2} \oplus \cdots \oplus \Delta_{n}>\cdots>\Delta_{n}>(0)$ for $R^{+}-N$ as an operator group for any distributively generating set $S$ of $R$. Hence we can use the Jordan-Hölder theorem for operator groups to show that any two composition series of $R$-subgroups of $R^{+}-N$ are of the same length and the factor groups of one of the series are $R$-isomorphic to the factor groups of the other series in some order. 
irreducible $R$-groups. We define a left ideal $N^{\prime}$ as follows:

$$
N^{\prime}=\cap\left\{\mathfrak{l}: \mathfrak{l} \text { is a left ideal and } R^{+}-\left\{\in I^{\prime}\right\}\right. \text {. }
$$

Clearly such left ideals $\backslash$ are maximal left ideals and so $N^{\prime} \geqq N$. It will be shown that some power of $N^{\prime}$ annihilates $A_{n}$ but, since $\Delta_{n}$ is faithful, this means that $N^{\prime}$ is a nilpotent left ideal. Hence $N^{\prime} \leqq N$ and consequently $N^{\prime}=N$. Thus $N$ is the intersection $\bigcap_{i=1}^{m} \mathfrak{l}_{i}$ of maximal left ideals $\mathfrak{l}_{i}$ of $R$ for which $R^{+}-\mathfrak{l}_{i} \in I^{\prime}$. We may assume that $\bigcap_{\substack{i=1 \\ i \neq j}}^{m} \mathfrak{l}_{i} \neq N$ for all $j=1, \ldots, m$. As indicated for the decomposition (1) of $R^{+}-N$, this leads to the decomposition $R^{+}-N=\Delta_{1}^{\prime} \oplus \cdots \oplus \Delta_{m}^{\prime}$, where $\Delta_{k}^{\prime} \cong R^{+}-\Upsilon_{k} \in I^{\prime}$ for $k=1, \ldots, m$. These $\Delta_{k}^{\prime}$ are cyclic irreducible $R$-groups. Thus both this decomposition and decomposition (1) provide us with composition series of $R^{+}-N$ and so $n=m$ and the $\Delta_{k}$ are $R$-isomorphic to the $\Delta_{j}^{\prime}$ in some order ${ }^{7}$ ). The theorem now follows from theorem 3 .

We shall prove that every group in $I$ is annihilated by some power of $N^{\prime}$ by induction on the order of the groups in $I$.

If $\Omega$ has least order among the non-zero groups in $I$, it is a minimal $R$-group and so is in $I^{\prime}$. Let $w \in \Omega, w \neq 0$; then $R w=\Omega$ and hence $R^{+}-(0: w) \triangleq \Omega$ so that the left ideal $(0: w)$ contains $N^{\prime}$. This is true for every element of $\Omega$ and consequently $N^{\prime}$ annihilates $\Omega$ itself.

Now let $\Omega \in I$ be an $R$-group of order $m>0$ and assume that all groups in $I$ of order less than $m$ are annihilated by some power of $N^{\prime}$. Let $\Omega=$ $\Omega_{0}>\Omega_{1}>\cdots>\Omega_{l}=(0)$ be a strictly descending chain of $R$-groups in $Q$ such that each $\Omega_{i}$ is normal in $\Omega_{i-1}$ and $\Omega_{i-1}-\Omega_{i}$ is an irreducible $R$-group (not necessarily cyclic). Each $\Omega_{i-1}-\Omega_{i}$ is contained in $I$. If $l>1$, then these factor groups have order less than $m$ and so, by hypothesis, are annihilated by some power of $N^{\prime}$. Therefore $\Omega$ itself is annihilated by some power of $N^{\prime}$. We are left with the case $l=1$, i.e., when $\Omega$ is an irreducible $R$-group. If we $\Omega$ and $R w=Q$, then $\Omega \in I^{\prime}$ and $R^{+}-(0: w) \bumpeq \Omega$. Therefore $(0: w)$ contains $N^{\prime}$ and hence $N^{\prime}$ annihilates $w$. If $w \in \Omega$ and $R w \notin \Omega$, then $R w \in I$ has order less than $m$ and by hypothesis $R w$, and so $w$, is annihilated by some power of $N^{\prime}$. Thus every element of $\Omega$ and therefore $\Omega$ itself is annihilated by some power of $N^{\prime}$. It follows, therefore, that every group in $I$, in particular $A_{x}$ itself, is annihilated by some power of $N^{\prime}$. This proves the first part of the theorem.

Now let $\Omega$ and $\Omega^{\prime}$ be two faithful, cyclic irreducible $R$-groups. They are finite groups. We have shown that $\Omega$ is an $R$-homomorphic image of an $R$-subgroup of $\Omega^{\prime}$ and, conversely, $\Omega^{\prime}$ is an $R$-homomorphic image of an $R$-subgroup of $\Omega$. Thus $\Omega$ and $\Omega^{\prime}$ are isomorphic.

Besides the properties of a d.g. near-ring described at the end of section 1 we have the following additional properties for a d.g. near-ring $R$ which satisfies the d.c.c. for left $R$-modules:

\footnotetext{
7) See footnote ${ }^{5}$ ).
} 
The radical $M$ of $R$ is an ideal containing all the nilpotent left $R$-modules of $R$ and the factor d.g. near-ring $R / M$ contains no non-zero nilpotent left $R / M$-modules, i.e., $R / M$ is semi-simple. The quasi-radical is a nilpotent left ideal containing all the nilpotent left ideals of $R$ (see [8]). The ideal-radical $P$ is a nilpotent ideal containing all the nilpotent ideals of $R$. Furthermore, the factor d.g. near-ring $R / P$ contains no non-zero nilpotent ideals.

\section{The nilpotency of the radical}

In $[8]$ we gave necessary and sufficient conditions for the radical of a d.g. near-ring to be nilpotent. We restate these conditions below and add new conditions in terms of prime ideals.

Theorem 6. Let $R$ be a d.g. near-ring satisfying the d.c.c. for left $R$-modules. The following conditions are equivalent:

(a) The radical is nilpotent.

(b) The radical is the quasi-radical.

(c) Every maximal left ideal is a maximal left $R$-module.

(d) The radical is the ideal-radical.

(e) Every proper prime ideal is maximal.

(f) Every cyclic irreducible $R$-group is a minimal $R$-group.

Proof. (a) implies (b). The radical contains quasi-radical and the quasiradical contains all the nilpotent left ideals of $R$. Therefore the radical is the quasi-radical if it is nilpotent.

(b) implies (c). If the radical $M$ is the quasi-radical, then every maximal left ideal of $R$ contains $M$. But $R / M$ is a semi-simple d.g. near-ring and so is a direct sum of left ideals of $R / M$ which are minimal left $R / M$-modules (see $[8]$ ). It follows that every maximal left ideal is a maximal left $R$-module.

(c) implies (d). If every maximal left ideal is a maximal left $R$-module, then the radical is the quasi-radical and so is a nilpotent ideal. But the radical contains the ideal-radical and the ideal-radical contains every nilpotent ideal of $R$. Therefore the radical is the ideal-radical if it is nilpotent.

(d) implies (e). If the radical $M$ is the ideal-radical, then every prime ideal of $R$ contains $M$. Let $\mathfrak{p}$ be a prime ideal of $R$. Then $m_{1} m_{2} \ldots m_{p} \subseteq m_{1} \cap m_{2} \cap \ldots$ $\cap \mathfrak{m}_{r}=M \leqq p$, where $m_{1}, \ldots, \mathfrak{m}_{r}$ are maximal ideals of $R$ (if $R$ satisfies the d.c.c. for left $R$-modules, then the radical is, in fact, an intersection of a finite number of maximal ideals). Hence $\mathrm{m}_{i} \leqq p$ for some $i$ and therefore $\mathrm{n}_{i}=\mathfrak{p}$.

(e) implies (f). Let $\Omega$ be a cyclic irreducible $R$-group. Then the annihilating ideal $\mathfrak{p}=(0: \Omega)$ is prime and is a maximal ideal if condition (e) is satisfied. In this case $R / p$ is a simple $\mathrm{d}$.g. near-ring and so is a direct sum of isomorphic minimal $R / p$-groups (see $[\boldsymbol{\gamma}]$ ). Since $\Omega$ is an $R / p$-group and has an $R / p$-generator it follows that it is a direct sum of minimal $R / p$-groups. But $\Omega$ is irreducible and so it must be a minimal $R / p$-group. Hence $\Omega$ is a minimal $R$-group. 
(f) implies (a). Let $\Upsilon$ be a maximal left ideal of $R$. Then $R^{+}-\Upsilon$ is a cyclic irreducible $R$-group and hence it is a minimal $R$-group if condition (f) is satisfied. Thus $I$ is a maximal left $R$-module. Therefore the radical and the quasi-radical must be equal in this case and therefore the radical is nilpotent.

\section{An example of a d.g. near-ring with a non-nilpotent radical}

We construct an example of a finite d.g. near-ring with a non-nilpotent radical and a quasi-radical which is not an ideal.

Let $\Omega$ be a finite, non-abelian, simple group and $R$ the near-ring generated by all the inner-automorphisms of $\Omega$. It has been shown in [4] and [ 7 ] that $R$ is a finite, simple d.g. near-ring with an identity element. If $\Delta$ is a subgroup of $\Omega,(\Delta: \Omega)$ is a right $R$-module and any right $R$-module $\mathfrak{r}=(\Delta: \Omega)$ for some subgroup $A$. There is a one-to-one lattice correspondence given by $r=$ $(\Delta: \Omega) \leftrightarrow \mathfrak{r} \Omega=\Delta$ between the right $R$-modules of $R$ and the subgroups of $\Omega$. Furthermore, right ideals and normal subgroups correspond to each other. Consequently, $R$ has proper, non-zero right $R$-modules but no proper, non-zero right ideals. Finally, we note that each right $R$-module $r$ has an element $e_{\mathfrak{r}}$ such that $\mathfrak{r}=e_{\mathfrak{r}} R$.

Now consider the near-ring $T$ generated by all the endomorphisms $\Phi_{x}$ of $R^{+}$, for all $x \in R$, where $\Phi_{x}(y)=y x$ for all $\left.y \in R^{+8}\right)$. It is clear that $T$ is a finite d.g. near-ring with an identity element; $R^{+}$is a faithful $T$-group and the identity element of $R$ is a $T$-generator of $R^{+}$. But the $T$-subgroups of $R^{+}$ are precisely the right $R$-modules of $R$ and so $R^{+}$is an irreducible $T$-group with a $T$-generator which is not a minimal $T$-group. It follows from theorem 5 that $T$ does not have a nilpotent radical.

Since $R^{+}$is faithful, the ideal radical of $T$ is the zero ideal ( $T$ is a finite, prime d.g. near-ring which is not simple). If the quasi-radical were an ideal, it would be the zero ideal (since the quasi-radical is nilpotent). But then $T$ is a direct sum of irreducible $T$-groups by the equality (1) after the corollary to theorem 2. Every $T$-subgroup $r$ of $R^{+}$has a $T$-generator $e_{r}$ and consequently every $T$-subgroup is a direct sum of irreducible $T$-subgroups. By the latticeisomorphism, this means that every subgroup of $\Omega$ is a direct sum of simple groups. If we take $\Omega$ to be the alternating group on nine symbols, then it has a cyclic subgroup of order nine and this subgroup is not a direct sum of simple subgroups, i.e., two subgroups of order three. It follows that the quasiradical of $T$ corresponding to the alternating group on nine symbols is not an ideal.

It is an open question whether or not the quasi-radical of a d.g. near-ring can be an ideal and yet not be the radical also. Or, to put it another way, whether a d.g. near-ring can possess non-zero nilpotent left $R$-modules and yet possess no non-zero nilpotent left ideals.

8) These mappings of $R^{+}$into itself are $\epsilon$ ndomolphisms because $R$ satisfies the right distributive law. 


\section{References}

[1] Bersch, G.: Ein Radikal für Fastringe. Math. Z. 78, 86-90 (1962).

[2] FröHLICh, A.: Distributively generated near-rings (I. Ideal theory). Proc. London Math. Soc. 8, 76-94 (1958).

[3] - Distributively generated near-rings (II. Representation theory). Proc. London Math. Soc. 8, 95-108 (1958).

[4] - The near-ring generated by the inner-automorphisms of a finite simple group. J. London Math. Soc. 33, 95-107 (1958).

[5] - On groups over a d.g. near-ring, Quart. J. of Math. (Oxford), (second series). (I) Sum constructions and free $R$-groups 11, 193-210 (1960); (II) Categories and functors 11, 211-228 (1960).

[6] Johnson, R. E.: Prime rings. Duke Math. J. 18, 799-809 (1951).

[7] LAxToN, R. R.: Primitive distributively generated near-rings. Mathematika 8, $142-158$ (1961).

[8] - A radical and its theory for distributively generated near-rings. J. London Math. Soc. 38, 40-49 (1963).

[9] McCoy, N. H.: Prime ideals in general rings. Amer. J. Math. 71, 823-833 (1949).

The University of Michigan, Mathematics Department, Ann Arbor, Mich., USA

(Received June 1, 1963) 